* Corresponding author E-mail address:stan kut@prz.edu.pl (Dr inż. Stanisław Kut)

Article information

Article history: AMS-Volume16-No.1-00144-12

Received 15 January 2012

Accepted 15 February 2012

\section{The analysis of the constitutive model effect on convergence of results with the experiment for FEM modeling of 3D issues with high elastic-plastic deformations}

\author{
Stanisław Kut \\ Rzeszów University of Technology, Faculty of Mechanical Engineering and Aeronautics, Poland
}

\section{BIOGRAPHICAL NOTES}

Stanisław Kut, Dr inż. was born in 1975 in Ropczyce, Poland. He received his MSc. Eng. and PhD. degree at Rzeszow University of Technology. Since 2004 he is an assistant professor at the Department of Materials Forming and Processing, Faculty of Mechanical Engineering and Aeronautics, Rzeszow University of Technology. He is autor of 2 monographies, one patent, 6 patent applications and more than 50 publications in scientific journals and conference proceedings.

\section{KEY WORDS}

tension, FEM modeling, constitutive relation, finite elastic-plastic deformation, ductile fracture

\begin{abstract}
This paper presents the results of experimental researches and FEM modeling for tension process of an original plane specimen with notch. The specimen was made of the low carbon steel sheet with thickness of $4 \mathrm{~mm}$. The modeling was performed with three different constitutive relationships, which are available in commercial software for modeling the non-linear issues with high strains. The three-dimensional numerical model was based on the experimental model. The results of individual models were compared with the experimental results. The special attention was paid to convergence of force characteristics and modeled specimen shapes in areas of the highest elastic-plastic deformations. Moreover, for the model of the highest convergence with the experiment the ductile fracture phase was modeled until total separation of specimen material.
\end{abstract}

\section{Introduction}

The numerical model parameters and the modeling itself have the important effect on convergence of achieved FEM modeling results with the experiment. This is especially important when modeling highly non-linear issues with high strains. Such issues are typical for modeling metal forming processes. The convergence of FEM modeling results with the experiment is influenced, among the others, by setting up the commercial software by the user:

- type, class, and number of finite elements in the model, 
- boundary and contact conditions,

-yield stress condition, which determines the three-dimensional state of stress corresponding to the beginning of the flow,

- the flow rule, which specifies the plastic strain increments,

- the strain hardening rule, which determines the plastifying condition modification during the material flow,

- the constitutive model, which determines the relation between stress and strain, etc.

The ability to modify of the above mentioned modeling parameters is the class proof of the commercial software used to process the non-linear and contact issues.

Large displacement and large strain inelastic response calculations are pursued to an increasing extent. Most commonly finite element procedures are used. An important basic ingredient in such a solution is the evaluation of the stresses, i.e. when the total strains have been calculated, it is necessary to evaluate the corresponding stresses and material internal variables [1].

In the infinitesimal theory an additive decomposition of the strain rate tensor is used, see Refs [2] and [3].

$D=D^{E}+D^{P}$

The deformation rate $\mathrm{D}$ is supposed to be decomposed as the sum of plastic (P) and elastic (E) parts.

Green and Naghdi [2] extended this approach to the finite deformation theory analysis. This formulation was studied by Bathe et al. [4-5] and it was shown that:

- the use of the logarithmic Hencky strain is necessary to ensure isochoric plastic flow,

- the shear modulus is a function of the plastic strain state for certain loading conditions.

Lee [6] introduced a new formulation, based on a conceptual stress relaxed intermediate configuration. If the complete motion of the body of interest is described by the multiplicative decomposition of deformation gradient $F$, then the motion from the initial to the 'stress free' configuration is given by the plastic part of the deformation gradient FP and the motion from this configuration to the final state is given by FE. Therefore we have:

$F=F^{E}+F^{P}$
Lee [6] enumerated the difference between the formulations (1) and (2) to be:

- The elastic and plastic parts of the displacements, respectively, are additive. Since the strains are nonlinear expressions in the displacements, they are in general not additive.

- The elastic part of the deformation gradient premultiplies FP and is only a function of the stress state, while the plastic part is also given by the history of plastic flow. The product (2) is not commutative in general, as are the strains in the additive decomposition.

Of course, for small elastic strains assumptions (1) and (2) are identical. The stresses are usually obtained by an integral based upon the constitutive equations. In this integration process we can identify two specific difficulties. Firstly, the material rigid rotations need to be integrated accurately, and, secondly, the integration should allow for the change in the principal axes of the stress tensor during each time step.

A completely different approach is based on using a "total formulation". In this case the criterion of "strong objectivity" is trivially satisfied, because the total stresses are obtained using the total strains, see for an early contribution using this approach Bathe et al. [7]. This approach is more reasonable from a physical point of view than the rate-type approach. The question of choosing an incremental stress measure does not require special attention and the formulation of an incrementally objective stress update algorithm is no longer necessary. For large strain elasto-plastic analysis using "a total formulation" Eterovic and Bathe [8] proposed an efficient algorithm using logarithmic strains to obtain the stress state for a given deformation gradient. The basic idea of this algorithm is to use a hyperelastic approach to determine the elastic predictor stresses exactly, and then a plastic relaxation process to obtain the final stress state. The applicability of this procedure depends on whether a kinematical decomposition of the deformation gradient into an elastic and plastic part is possible. Furthermore, the plastic evolution equation has to be integrated so that the stress state is obtained from the elastic deformation state at the end of every time step.

This paper examined the effect of the constitutive model on convergence of modeling results with the experiment conducted. The analysis was 
performed for three models with the Lagrange formulation, implemented in commercially available software MSC MARC/Mentat 2010 [9]:

\section{- model (I) - total Lagrangian formulation,}

- model (II) - updated Lagrangian formulation, based on additive decomposition of deformation gradient,

- model (III) - updated Lagrangian formulation, based on multiplicative decomposition of deformation gradient.

The total Lagrangian procedure [9] can be used for linear or nonlinear materials, in conjunction with static or dynamic analysis. Although this formulation is based on the initial element geometry, the incremental stiffness matrices are formed to account for previously developed stress and changes in geometry. The total Lagrangian approach is also useful for problems in plasticity and creep, where moderately large rotations but small strains occur. A case typical in problems of beam or shell bending. However, this is only due to the approximations involved.

The updated Lagrangian approach [9] is useful in analysis of shell and beam structures in which rotations are large so that the nonlinear terms in the curvature expressions may no longer be neglected, and large strain elasticity and plasticity analysis. For large strain elastic-plastic analysis, the default procedure in Marc uses a procedure based on an additive decomposition of incremental strain into an elastic part and a plastic part, together with a mean normal return-mapping algorithm. In this case, volumetric strain in a lower-order plane strain, axisymmetric or 3-D brick element is assumed to be constant for von Mises plasticity to overcome volumetric locking because of the possible large and incompressible plastic deformation. Marc can also use a procedure based on a multiplicative decomposition of deformation gradient into an elastic part and a plastic part together with a radial return mapping algorithm for large strain elastic-plastic analysis. A mixed formulation is used to deal with the problem associated with volume constraints. This procedure is only available for continuum elements.

\section{Experimental Procedure}

The researches have been conducted on the specimens prepared as follows: sheet strips, width of $16 \mathrm{~mm}$, length of $100 \mathrm{~mm}$, and thickness of
$4 \mathrm{~mm}$, along the rolling direction. The specimen notch has been prepared using the milling cuter on depth of $(2 / 3) \mathrm{g}$, where $\mathrm{g}$ is sheet thickness. The tool and specimen preparation method and shape with dimensions have been presented on Fig. 1.

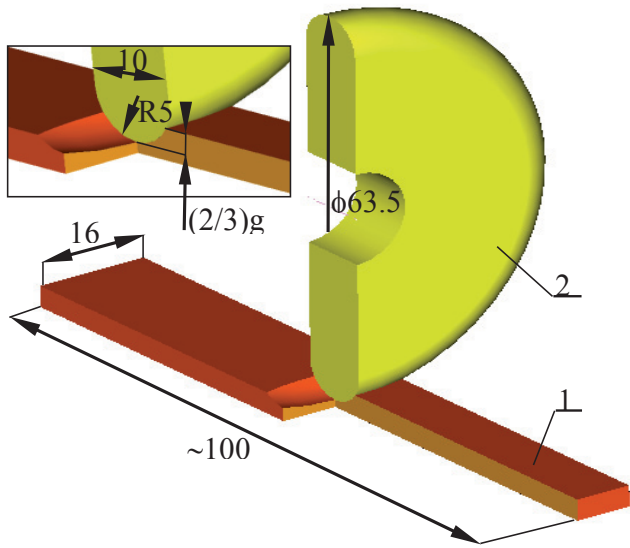

Fig. 1: The notch on the specimen: 1 - specimen and 2 - milling cuter.

The examined material mechanical properties (Table 1) have been determined based on the typical tension test conducted on tensile testing machine UTS 100, with the extensometer measuring base of $80 \mathrm{~mm}$.

Table 1: Mechanical properties of materials tested.

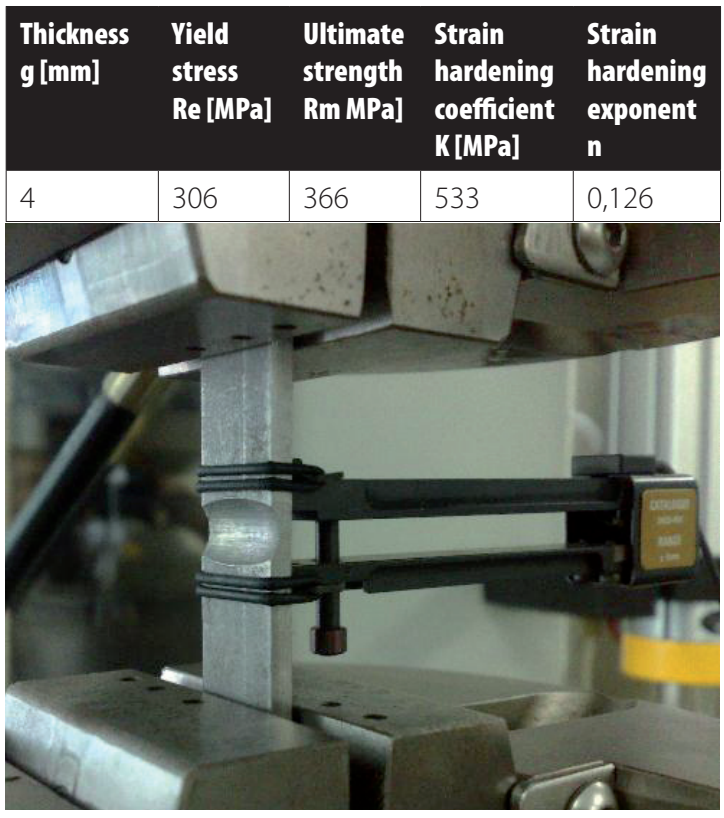

Fig. 2: The specimen under tension test. 


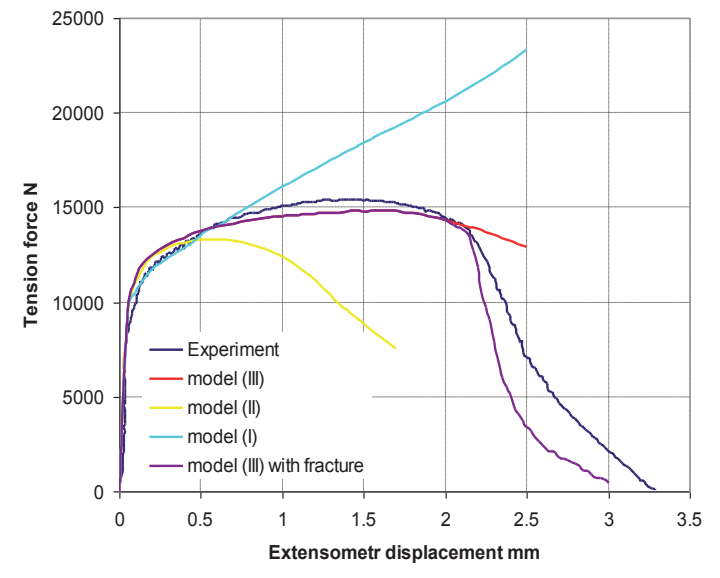

Fig. 3: Tension process force characteristics.

The experimental tension test of three specimens with notch has been performed using the tensile testing machine Instron 8801. The extensometer with the measuring base of $12 \mathrm{~mm}$ has been fixed to the specimen (Fig. 2). The test was performed along with capturing the force characteristics until the specimen breach. The mean extensometer displacement for fracture initiation has been determined based on captured force characteristics (example on Fig. 3). This value was $2.15 \mathrm{~mm}$.

After tension tests, the torn specimens were measured using the toolmaker's microscope Nikon MM-800/L with DS-Vi1 camera and software. The specimen thickness was measured in a fracture area, in the middle and on sides. The average measured specimen thickness (Fig. 4) in its middle part was $0.89 \mathrm{~mm}$, and on side was $2.05 \mathrm{~mm}$. Afterwards the flank profile of deformed specimen was mapped by measuring the coordinates of points falling on its line. The shape and dimensions of measured flank outline were presented on Fig. 5.

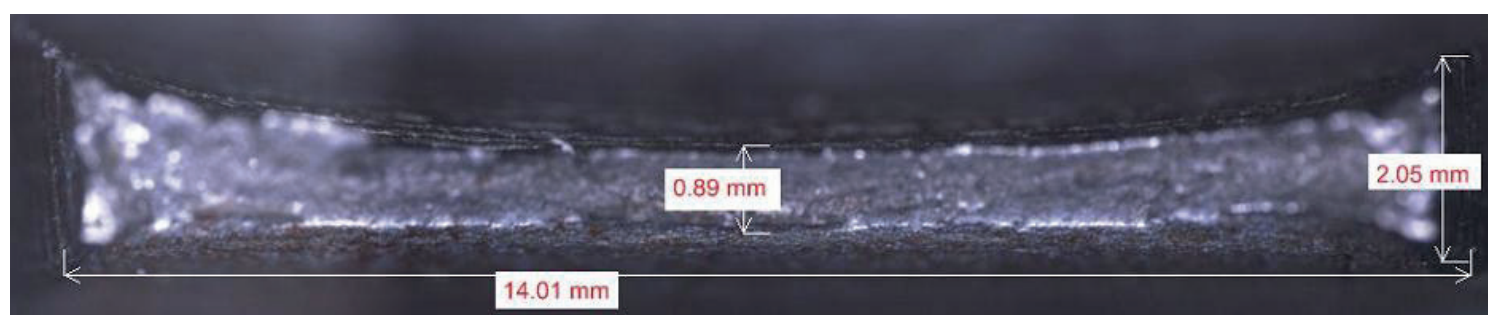

Fig. 4: The fracture surface with indicated average measured values.

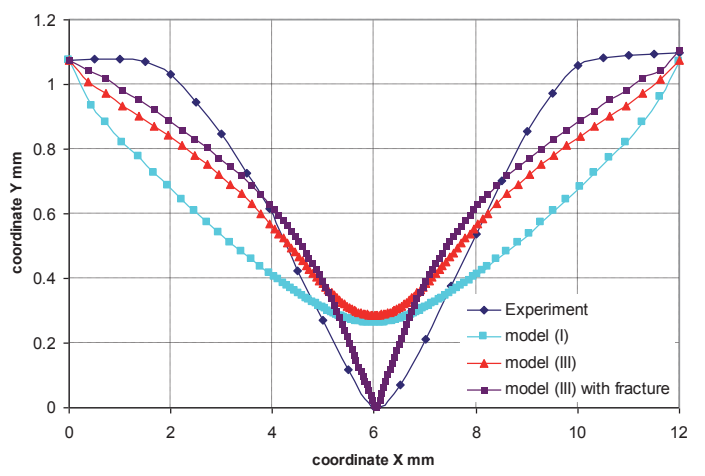

Fig. 5: The profile of specimen flank.

\section{Finite Element Modeling}

The FEM model of the tension test has been created based on the experimental model. As the examined specimen has a symmetry in its plane, three-dimensional geometrical model of the specimen half has been created. This reduced the required computing power. The three-dimensional eight-node hex 8 type 7 elements have been used in order to digitize the model [10]. The element dimension was from $\sim 0.25 \mathrm{~mm}$ to $\sim 0.05 \mathrm{~mm}$ in the highest strain area. The tension process has been modelled from the beginning of fracture, determined during the experiment.

The material properties has been described using the elastic-plastic model with Huber-Mises plastifying function and isotropic hardening. The flow rule has been described using the Prandtl-Reuss representation. This is one of typical models used in MARC software.

The material parameters for elastic strain have been as follows: modulus of elasticity $E=210000$ $\mathrm{MPa}$, Poisson number $v=0.3$. The relation between strain and logarithmic strain within the plastic strain region has been entered to the software as a table according to the strain hardening curve course, approximated using the power equation (Hollomon's equation). The parameters for the strain hardening curve are presented in Table 1. 
The modeling was performed for three constitutive models as described at the beginning (model I - total Lagrangian, model II — additive decomposition, model III - multiplicative decomposition). Other modeling parameters were identical for analyzed models.

All models were analyzed in extent of elasticplastic deformation to the beginning of fracture, which was determined experimentally. Moreover, for model III, which featured the highest convergence of results with the experiment, the modeling was also extended by fracture phase. For this purpose, the customized subroutine for fracture phase modeling was developed and implemented in commercial software MARC/Mentat. Rice and Tracey model was used as a ductile fracture criterion [11]:

$$
\int_{0}^{\varepsilon f} \exp \left(1.5 \frac{\sigma_{m}}{\sigma_{e}}\right) d \varepsilon_{e q}=C
$$

where: $\sigma_{m}$ mean normal stress, $\sigma_{e}$ effective stress, $\varepsilon_{f}$ fracture strain, $\varepsilon_{e q}$ equivalent plastic strain.

The material constant value $\mathrm{C}=1.93$ was determined by reversed method. In this case, this method comprised of modeling notched specimen tension process and calculating the integral value in individual calculation iterations until the beginning of fracture, determined during the experiment. The maximum value of calculated integral in the moment of beginning of fracture in the experiment is the unknown value of $C$.

The fracture phase modeling procedure was operated as follows:

(1) in each iteration i for each element $j$, the subroutine gets the following data from the source program $\sigma_{m_{j}}, \sigma_{e_{j}}, \varepsilon_{e q j}$.

(2) for each element in each analysis step, the so called fracture potential is calculated:

$D_{f}=\frac{\int_{0}^{\varepsilon f} \exp \left(1.5 \frac{\sigma_{m}}{\sigma_{e}}\right) d \varepsilon_{e q}}{C}$

(a)If $D_{f_{i j}}<1$, the program goes to the next iteration.

(b) If $D_{f_{i j}} \geq 1$, the program subroutine deletes this item and then goes to the next iteration.

(3) The cycle repeats.

Thus during the ductile fracture phase modeling, successive elements are deleted and the gap is cre- ated, which emerges in the direction of maximum fracture potential until total separation of formed material.

\section{Results and Discussion}

The comparison of force characteristics is one of the basic methods for process modeling used when calibrating and validating numerical models against the experiment. In real environment, such a calibration comprises of numerical model and modeling parameter selection to achieve the satisfactory characteristics convergence. In this case, the constitutive model selection significantly influences the force characteristics comparing to the experiment. When observing the force characteristics (Fig. 3), the highest convergence for small elastic-plastic deformations are visible in models (II) and (I). Model (III) tends to slightly higher force values in this range. For higher deformation values, the calculated force characteristics for individual models become more and more different between each other. For high deformations preceding the fracture, these differences are very high, especially between model (II) and (I). In this case, the force values may be highly reduced (model II) or highly increased (model I). Considering the force characteristics in a whole deformation range until moment of fracture, model (III) features the highest convergence with the experiment. For low deformations, this model features slightly increased values, and for higher deformations slightly reduced values. For deformations preceding the fracture, model (III) featured good convergence with the experiment. As for the model (II), the necking locating became quite faster than for a real experiment, this process was not modeled to value of measuring base displacement of $2.5 \mathrm{~mm}$, but for $1.75 \mathrm{~mm}$. This caused by a fact that for displacement of 1.75 $\mathrm{mm}$, the specimen necking in the middle part was about 95\%.

I many cases, the accuracy of achieved material shape is very important modeling result, especially in an area of high elastic-plastic deformations and once the material is separated. For an accurate comparison of these parameters, the specimen flank profiles and an experimentally achieved profile were presented on the chart (Fig. 5). The profiles were measured on specimen length $X$ of measuring base of $12 \mathrm{~mm}$. Y coordinate was measured on the deformed specimen flank in order to determine its outline. As the model (II) featured the 
necking earlier than the experiment, this model was disregarded in this comparison. Obviously for modeling without material separation, model (I) and (III) significantly differ from the experiment in an area of the highest deformation for $X=6 \mathrm{~mm}$. However, both models feature similar results in this range. In other area, model (III) features better results. Even better results may be achieved by including the ductile fracture phase until material separation in the modeling. In this case, for model (III) with fracture the highest convergence was achieved, especially in the highest necking on the specimen width. The thickness change of deformed specimen is another parameter influencing the modeling effectiveness. The specimen thickness $t$ was measured in its thinnest area. For nondeformed specimen $\mathrm{t}=1.35 \mathrm{~mm}$. The specimen thickness $t$ reduces as the tension process progesses (Fig. 6). For model (II), the thickness reduction is too fast due to a fast necking location. Thickness $\mathrm{t}=0.89 \mathrm{~mm}$ was achieved for displacement of extensometer of $0.76 \mathrm{~mm}$, whereas in real environment the displacement was about $2.15 \mathrm{~mm}$. Much better results were achieved for model (I), which also forecasts slightly higher specimen thickness reduction than for the experiment. The best convergence was achieved for model (III), which is practically identical with the experimental value in moment of beginning of fracture.

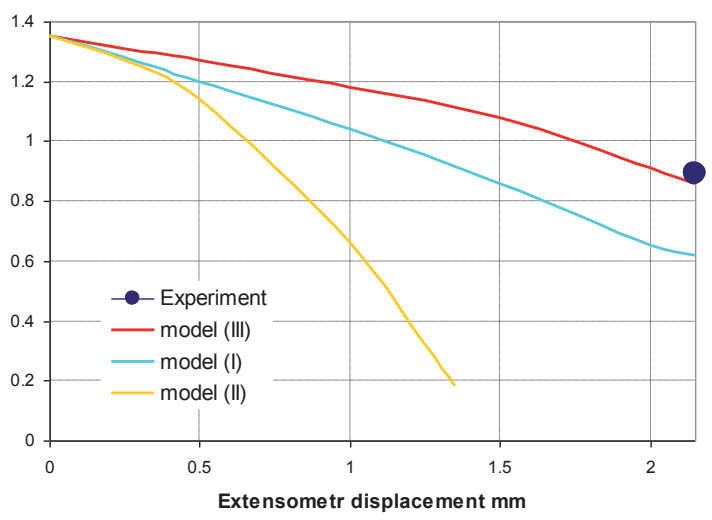

Fig. 6: The specimen under tension test.

Considering the ductile fracture phase in modeling enables various capabilities when forecasting ductile fracture, i.e. forecasting the specimen shape once the material is separated. Figure 7 presents the specimen after the tension process. Both for the experiment (Fig. 7a) and for FEM simulation (Fig. 7b), the fracture initiation occurs in the middle part of specimen and during further tensioning the gap develops until total material separation. When comparing the specimen shape and visible gap in the sheet plane and the shape of separation surface, it is clearly visible that model (III) including the ductile fracture phase and Rice and Tracey criterion enabled achieving good convergence of FEM modeling results with the experiment.

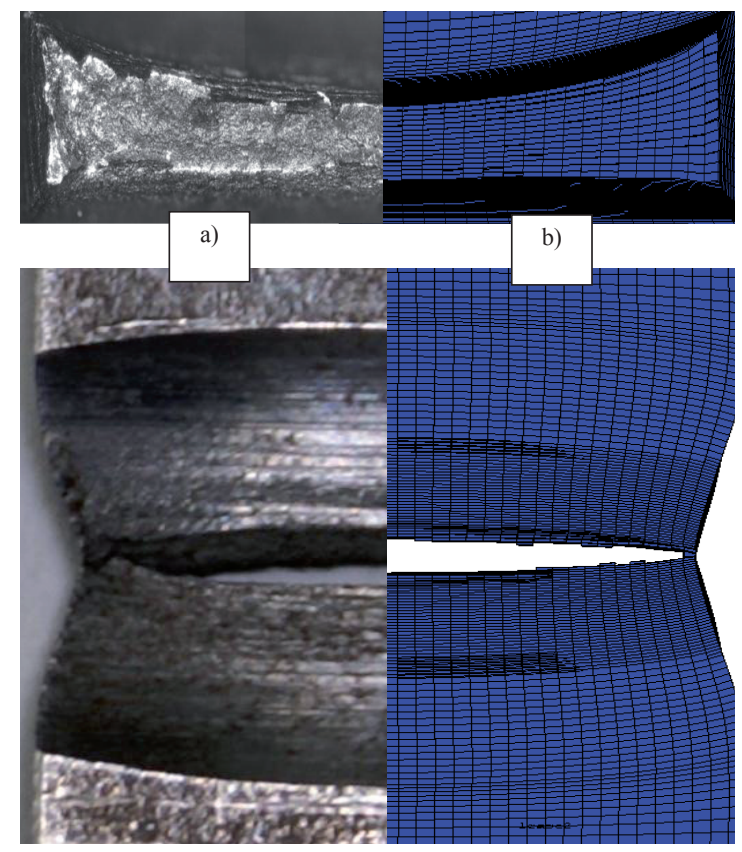

Fig. 7: Broken specimen view: a) experiment, b) FEM simulation

\section{Conclusion}

The performed researches revealed that the highest convergence of modeling results with the experiment for 3D modeling of plane specimen with notch tension process was achieved for model (III). This model enabled achieving the best results for modeling of elastic-plastic issues with high deformations both from point of view of force characteristics and shape mapping correctness. Considering the fracture phase until material separation enabled achieving fair results in form of not only convergent force characteristics, but also shape and dimensions of specimen in highly deformed fracture area.

\section{Acknowledge}

The researches were performed using the R\&D devices purchased within the project POPW.01.03.00- 
18-012, the Structural Funds of Development of Eastern Poland Operational Programme, co-financed by European Union with European Regional Development Fund.

\section{References}

[1] Bathe K.J., Finite Element Procedures, Prentice Hall, Englewood Cliffs, NJ 1995.

[2] Greek A.E., Naghdi P.M., A general theory of an elastic-plastic continuum, Arch. Rat. Mech. Anal. 18, 1965, p. 251-281.

[3] Gabriel G., Bathe K. J., Computational issues in large strain elasto-plastic analysis, Computers\&Structures, Vol. 56, No. 2/3, 1995, p. 249-267.

[4] Bathe K.J., Kojić M., Walczak J., Some developments in methods for large strain elasto-plastic analysis, Computational plasticity: Models, Software and Applications (Edited by D. J. R. Owen et al.), Pineridge Press, Swansea, 1987, p.263-279.

[5] Eterovic A.L., Bathe K.J., A note on the use of the additive decomposition of the strain tensor in finite deformation inelasticity, Comput. Meth. Appl. Mech. Engng 93,1991, p. 31-38.

[6] Lee E.H., Elastic-plastic deformation at finite strains, J. Appl. Mech. 36,1969, p. 1-6.

[7] Bathe K.J., Ramm E., Wilson E.L., Finite element formulations for large deformation dynamic analysis, Int. J. Numer. Meth. Engng 9, 1975, p. 353-386.

[8] Eterovic A.L., Bathe K.J, A hyperelastic-based large strain elasto-plastic constitutive formulation with combined isotropic-kinematic hardening using the logarithmic stress and strain measures, Int. J. Numer. Meth. Engng 30, 1990, p. 1099-1114.

[9] MSC Software, MSC.Marc Volume A, Theory and User Information, Version, 2010.

[10] MSC Software, MSC.Marc Volume B, Element Library, Version, 2010.

[11] Rice J.R., Trecey D.M., On the ductile enlargement of voids in triaxial stress fields, J. Mech. Phys. Solids 17, 1969, p. 201217. 